\title{
REVIEW \\ Definition and management of ruxolitinib treatment failure in myelofibrosis
}

\author{
A Pardanani and A Tefferi
}

Ruxolitinib, a Janus kinase (JAK)-1 and JAK-2 inhibitor, is the first-in-class drug to be licensed in the United States for the treatment of high- and intermediate-risk myelofibrosis (MF). Several other JAK inhibitors are in development with some currently undergoing phase-3 clinical trial testing. None of the currently available JAK inhibitors are specific to mutant JAK2; their mechanism of action involves attenuation of JAK-STAT signaling with downregulation of proinflammatory cytokines, rather than selective suppression of the disease clone. Accordingly, while ruxolitinib and other JAK inhibitors are effective in controlling splenomegaly and alleviating constitutional symptoms, their benefit in terms of reversing bone marrow fibrosis or inducing complete or partial remissions appears to be limited. The experience to date with ruxolitinib shows that despite its salutary effects on quality of life, over half of the patients discontinue treatment within 2-3 years. In the current perspective, we examine the incidence and causes of ruxolitinib 'treatment failure' in MF patients based on our personal experience as well as a review of the published literature. We also discuss the challenges in defining and classifying ruxolitinib failure, and within the context of several clinical scenarios, we provide recommendations for the post-ruxolitinib management of MF patients.

Blood Cancer Journal (2014) 4, e268; doi:10.1038/bcj.2014.84; published online 12 December 2014

\section{SCOPE OF THE PROBLEM}

Clinical manifestations, natural history and prognosis of myelofibrosis

The discovery of the JAK2V617F mutation, ${ }^{1}$ and subsequently, other Janus kinase (JAK)-STAT pathway activating mutations in myeloproliferative neoplasms, ${ }^{2,3}$ provided the early rationale for the development and use of JAK inhibitors as a treatment modality for these conditions, in particular for myelofibrosis (MF) given its relatively poor prognosis as compared with polycythemia vera or essential thrombocythemia. ${ }^{4}$ Patients with MF are highly symptomatic, and present with marked splenomegaly (31\%), anemia often requiring red blood cell transfusions (38\%) and constitutional symptoms (34\%). ${ }^{5}$

The natural history of primary MF (PMF) has now been relatively well defined; analysis of mature data sets such as that recently reported from the Mayo Clinic (64\% deaths, median follow-up for living patients $=7.7$ years) revealed a median survival of 5.9 years from the time of referral to that center and an approximately $7 \%$ leukemic transformation rate. ${ }^{4}$ PMF patients can be risk stratified in terms of survival on the basis of either clinical risk models such as the Dynamic International Prognostic System-plus, ${ }^{6}$ or increasingly, on the basis of the underlying myeloproliferative neoplasms-relevant mutational profile. ${ }^{4,7-9}$ In terms of the latter, the presence of type 1/type 1-like CALR exon 9 mutations is associated with the most favorable survival outcome while the absence of JAK2/MPL/CALR mutations (that is, 'triple negative') is associated with the most unfavorable outcome. ${ }^{4}$ Furthermore, the presence of increasing number of prognostically detrimental//high molecular risk' mutations (that is, ASXL1, EZH2, SRSF2 and/or $I D H-1 / 2$ genes) conferred a progressively worse survival outcome, independent of traditional risk factors. ${ }^{7}$

\section{TREATMENT OF MF BEFORE THE ADVENT OF JAK INHIBITORS}

Drug therapy for PMF is not curative and has not been shown to display disease-modifying activity. ${ }^{10}$ Therefore, allogeneic stem cell transplant (ASCT) is often considered if the goal of therapy is to prolong survival. ${ }^{11}$ ASCT for PMF is potentially curative but its value has been undermined by treatment-related complications, including death. In general, asymptomatic patients with low/ intermediate-1 risk MF can be observed without specific treatment or with palliative therapy; androgens, prednisone, danazol, thalidomide and lenalidomide are used for the treatment of anemia and hydroxyurea for splenomegaly. In addition, splenectomy might be considered for drug-refractory splenomegaly and involved field radiotherapy for post-splenectomy hepatomegaly and non-hepatosplenic extramedullary hematopoiesis.

\section{DEVELOPMENT OF JAK INHIBITORS FOR MF}

Synopsis of the ruxolitinib story

JAK inhibitors for treatment of MF, including PMF and postpolycythemia vera or post-essential thrombocythemia MF, were first introduced in the form of INCB018424 (Ruxolitinib), a JAK-1/2 inhibitor, in a phase $1 / 2$ study (NCT00509899). On the basis of promising preliminary results in terms of treatment-induced reduction in splenomegaly and improvement in MF-related symptoms (dose limiting toxicity was reversible thrombocytopenia; maximum tolerated dose was $25 \mathrm{mg}$ twice daily or $100 \mathrm{mg}$ once daily), ${ }^{12}$ ruxolitinib was rapidly evaluated in two phase 3 registration trials, namely COMFORT-I (NCT00952289) and COMFORT-II (NCT00934544).

COMFORT I was a randomized double-blind placebo-controlled trial in patients with intermediate-2 or high-risk MF with palpable 
splenomegaly; the ruxolitinib starting dose was $20 \mathrm{mg}$ or $15 \mathrm{mg}$ twice daily based on the pre-study baseline platelet count. ${ }^{13}$ Unblinding of the study-drug assignments and crossover from placebo to ruxolitinib was permitted for protocol-defined worsening splenomegaly ( $\geqslant 25 \%$ increase in spleen volume from baseline). Of the 155 patients randomized to ruxolitinib, $41.9 \%$ achieved the primary end point (that is, $\geqslant 35 \%$ reduction in spleen volume by magnetic resonance imaging or computed tomography at week 24 as compared with baseline) versus $0.7 \%$ in the placebo arm $(P<0.001)$. Similarly, a greater proportion of patients on the ruxolitinib arm (45.9\%) versus placebo (5.3\%) met the key secondary end point of $\geqslant 50 \%$ decrease in the 6 item total symptom score (derived from a modified MF Symptom Assessment Form) $(P<0.001)$.

In COMFORT-Il, patients were randomly assigned, in a 2:1 ratio, to receive ruxolitinib or best available therapy (BAT), the latter including any commercially available agents or no therapy $(47 \%$ received hydroxyurea, $16 \%$ glucocorticoids and $33 \%$ no therapy). ${ }^{14}$ Patients receiving BAT who met protocol-specified criteria (underwent splenectomy or had an increase in spleen volume of $>25 \%$ from the nadir during the study period) could crossover to receive ruxolitinib therapy. Of the 146 patients randomized to ruxolitinib, $28 \%$ achieved the primary end point (that is, $\geqslant 35 \%$ reduction in spleen volume at week 48 as compared with baseline) versus $0 \%$ in the BAT arm $(P<0.001)$. Similarly, a greater proportion of patients on the ruxolitinib arm (32\%) versus placebo $(0 \%)$ met the key secondary end point of $\geqslant 35 \%$ reduction in spleen volume at week 24 as compared with baseline. On the basis of the aforementioned data, ruxolitinib was licensed for treatment of intermediate- and high-risk MF in the United States in $2011 .{ }^{15}$

\section{WHAT IS THE INCIDENCE OF RUXOLITINIB TREATMENT FAILURE?}

In the absence of a clearcut definition, the rate of ruxolitinib treatment discontinuation may provide an indirect estimate of treatment failure, although this proxy does not distinguish between 'true treatment failure' versus discontinuation for other reasons (for example, loss of insurance coverage and onset of a severe comorbid illness). In COMFORT-I and COMFORT-II, ruxolitinib discontinuation rates were approximately $50 \%$ by 3 years. ${ }^{16,17}$ However, another study based on US claims database inquiry suggested much higher rates (up to 50\% within 6 months) of discontinuation in routine clinical practice. ${ }^{18}$ Our experience is similar to the latter report; in a phase 1/2 study (NCT00509899), ruxolitinib discontinuation rates among 51 patients treated at our site at 1,2 and 3 years were 51,72 and $89 \%$, respectively. ${ }^{19}$ Conversely, ruxolitinib discontinuation rates for 107 patients treated at the other study site at 1, 2 and 3 years were 24, 36 and $46 \%$, respectively. ${ }^{20}$

\section{POTENTIAL CAUSES OF RUXOLITINIB TREATMENT FAILURE IN MF}

Lessons from phase 3 clinical trials

Resistance

Primary resistance (refractoriness): Most MF patients achieve at least some degree of spleen size reduction with ruxolitinib therapy; however, the minimum degree of splenic response that confers therapeutic benefit remains undefined. ${ }^{13,14}$ In contrast, while most patients randomized to ruxolitinib in COMFORT-I had at least some improvement in symptoms at week 24 as compared with baseline, a small but not insignificant proportion (not quantified in the manuscript) had worse symptoms at this time point. $^{13}$

Secondary resistance (Splenic relapse): COMFORT-I and COMFORT-II defined 'loss of spleen response' as spleen volume that was no longer $\leqslant 35 \%$ reduced as compared with baseline and increased by $\geqslant 25 \%$ from the nadir. The cumulative spleen response $\geqslant 35 \%$ reduction in spleen volume) at last follow-up was 59 and $51 \%$ for patients randomized to ruxolitinib in COMFORT-I and COMFORT-II, respectively. ${ }^{16,17}$ By 3 years, the median duration of spleen response by the aforementioned criteria had not been reached.

Treatment-related toxicities. The rate of study discontinuation due to adverse events (AEs) was similar between the two study arms in both COMFORT-I and COMFORT-II. ${ }^{13,14}$ Non-hematological toxicities (mostly grades $1 / 2$ in severity) were also comparable; however, ecchymosis, dizziness, diarrhea and headache occurred more frequently in patients randomized to ruxolitinib. Cytopenias were observed more frequently in patients treated with ruxolitinib as compared with placebo or BAT. In COMFORT-I, rates of grades $3 / 4$ anemia were 45 versus $19 \%$, thrombocytopenia 13 versus $1 \%$ and neutropenia 7 versus $2 \% .^{13}$ The mean hemoglobin level nadir $(9.5 \mathrm{~g} / \mathrm{dl}$, from $10.9 \mathrm{~g} / \mathrm{dl}$ at baseline) and highest red blood cell transfusion rate $(40 \%$, from $25 \%$ at baseline) were seen at 8 12 weeks after starting ruxolitinib treatment, with partial recovery by week $24 .^{13,14}$ In contrast, the mean platelet count decreased by approximately $50 \%\left(320\right.$ to $\left.170 \times 10^{9} / \mathrm{l}\right)$ in patients randomized to ruxolitinib, without recovery over time. ${ }^{17}$ Of note, both studies mandated modifications in the ruxolitinib dose in response to thrombocytopenia or neutropenia; in general, patients randomized to $20 \mathrm{mg}$ and $15 \mathrm{mg}$ twice daily were titrated down to $15 \mathrm{mg}$ and $10 \mathrm{mg}$ twice daily, respectively. ${ }^{16,17}$ In COMFORT-II, $63 \%$ of ruxolitinib versus $15 \%$ of BAT patients required dose reduction or interruption for $\mathrm{AEs}$, particularly for thrombocytopenia in the ruxolitinib arm (41 versus $1 \%) .{ }^{14}$

Disease progression, including leukemic transformation. In COMFORT-I, two patients and zero patient in the ruxolitinib and placebo arms, respectively, developed acute myeloid leukemia (AML) per the initial report. ${ }^{13}$ By 3 years, four patients in each arm had developed AML. ${ }^{17}$ In COMFORT-II, after a median follow-up of 151 weeks, five (3\%) and three (4\%) patients developed $A M L$ in the ruxolitinib and BAT arms, respectively. ${ }^{16} \mathrm{~A}$ recent report indicated a relatively high incidence of extramedullary $A M L$ in ruxolitinib-treated patients. ${ }^{21}$ Among 40 patients treated over 2 years, 5 patients $(13 \%)$ developed $\mathrm{AML}$, of which 4 patients developed isolated extramedullary leukemia.

Withdrawal symptoms on temporary or permanent drug discontinuation. Among patients who interrupt or discontinue ruxolitinib treatment, MF symptoms relapse to baseline levels in approximately 1 week. ${ }^{13}$ In COMFORT-I, AEs of $\geqslant$ grade 3 were reported in 16 and $57 \%$ of patients after ruxolitinib interruption and discontinuation, respectively (versus 13 and $46 \%$ in placebo arm). ${ }^{13}$ While no clear pattern of AEs after discontinuing ruxolitinib was recognized in either COMFORT-I or COMFORT-II, others have reported serious AEs manifesting as respiratory distress, shock, capillary leak, tumor lysis syndrome, splenic infarction and/or disseminated intravascular coagulation with fibrinolysis (DIC/ICF) in this setting. ${ }^{22-24}$ On occasion, withdrawal events may occur even in the context of preemptive measures such as tapering of ruxolitinib dose and administration of concurrent corticosteroid and/or myelosuppressive (for example, hydroxyurea) therapies. Severe AEs have been reported in some, ${ }^{25}$ but not other studies, ${ }^{26,27}$ in the context of pre-planned ruxolitinib discontinuation before ASCT; in one study, 7 of 10 patients developed serious AEs ( 7 were life-threatening, 2 fatal) within 21 days of discontinuation. ${ }^{25}$ Events of cardiogenic shock, tumor lysis syndrome/acute renal failure and steroid-refractory acute graftversus-host disease were reported.

Early mortality. COMFORT-I showed a survival benefit for patients randomized to ruxolitinib versus placebo after a median follow-up 
of 51 weeks (13 $(8 \%)$ versus 24 deaths $(16 \%)$; hazard ratio $=0.5$, $95 \%$ confidence interval $(\mathrm{Cl})=0.25-0.98){ }^{13}$ After a median followup of 149 weeks however, this survival advantage was lost (42 $(27 \%)$ versus 54 deaths (35\%); hazard ratio $=0.69,95 \% \mathrm{Cl}=0.46-$ 1.03). ${ }^{17}$ In contrast, COMFORT-II showed no survival advantage for ruxolitinib versus BAT after a median follow-up of 61 weeks (11 (8\%) versus 4 deaths (5\%); hazard ratio $=1.01,95 \% \mathrm{Cl}=0.32-$ 3.24). ${ }^{14}$ After a median follow-up of 151 weeks however, a survival advantage for ruxolitinib versus BAT was reported (29 (20\%) versus 22 deaths (30\%); hazard ratio $=0.48,95 \% \mathrm{Cl}=0.28-0.85){ }^{16}$

These analyses are confounded by the study design that permitted treatment crossover. For instance, in COMFORT-I, a high proportion of patients in the placebo arm either discontinued treatment $(27 \%)$ or crossed over to receive ruxolitinib early in the study (73\%; median time to crossover $=41$ weeks). Further, higher treatment discontinuation rates have been reported for patients randomized to ruxolitinib versus the comparator arms for both COMFORT-I and COMFORT-II studies. ${ }^{28,29}$ This may introduce a selection bias that cannot be compensated either by subject randomization or by intent-to-treat survival analyses.

\section{DEFINING RUXOLITINIB TREATMENT FAILURE IN MF}

Defining ruxolitinib failures in the context of routine clinical practice Currently, there is no consensus on what specific criteria define failure versus suboptimal response to ruxolitinib treatment in MF. In this regard, routine clinical practice remains at significant variance from conduct of clinical trials; specifically, imaging studies and symptom assessment questionnaires for assessment of spleen and symptom response, respectively, are not routinely utilized. The FDA label for ruxolitinib provides limited guidance in terms of dose reductions for thrombocytopenia, potential drugdrug interactions and impaired liver and/or kidney function, and also for dose increases mainly for inadequate spleen response. ${ }^{15}$

Defining ruxolitinib failures in the context of clinical trials

Development of other JAK inhibitor drugs is currently ongoing, including several in clinical trials that target patients who have received prior ruxolitinib therapy. This constitutes an attractive niche for drug development given the current lack of FDAlicensed drugs for the ruxolitinib 'relapsed/refractory' setting. Given the absence of a standard definition for ruxolitinib failure, several ad hoc solutions have been implemented in postruxolitinib clinical trials.

Simply require prior ruxolitinib exposure (without mandating that any pre-defined criteria for ruxolitinib failure be met). In the JAKARTA-2 study (NCT01523171), eligibility criteria for 'ruxolitinib failure' required prior exposure to ruxolitinib for at least 14 days. ${ }^{30}$ While this approach is practical and relatively unbiased, it relies entirely on physician/investigator judgment in terms of assigning whether a patient has failed ruxolitinib treatment. The basis of ruxolitinib failure in this context can be expected to be nonuniform and the underlying patient heterogeneity could complicate efforts to understand which aspects of ruxolitinib failure are amenable to being favorably modified by second-line therapies.

Specify study-specific criteria for ruxolitinib failure. In the ongoing phase 3 study of momelotinib versus BAT in MF (NCT02101268), eligibility criteria include prior exposure to ruxolitinib for at least 28 days with either a requirement for red blood cell transfusions while on ruxolitinib treatment or a need to adjust dose of ruxolitinib to $<20 \mathrm{mg}$ twice daily for either $\geqslant$ grade 3 thrombocytopenia or anemia or hematoma. While this approach provides a more specific definition of ruxolitinib failure, a potential disadvantage is that it is study specific and excludes certain patient subgroups who could also reasonably be considered as having failed ruxolitinib treatment (see examples below). Further, such definitions can be biased toward a patient population considered more likely to respond to the second-line therapy being tested, so as to increase the probability of a positive study outcome.

\section{CLINICAL SCENARIOS ILLUSTRATING FAILURE OF FIRST-LINE RUXOLITINIB TREATMENT AND OUR PROPOSED MANAGEMENT OPTIONS FOR EACH SCENARIO}

\section{Early-onset cytopenias: treatment-related myelosuppression}

a. Generally seen within the first 12 weeks of treatment.

b. Dose dependent to a large extent.

c. Lower baseline blood counts predispose to more severe treatment-emergent cytopenias.

d. Generally reversible with dose reduction or treatment interruption.

\section{Management options:}

a. Support with red blood cell/platelet transfusions as necessary.

b. Decrease dose or interrupt treatment depending upon severity of cytopenias. Treatment with a lower dose can be resumed after blood count recovery.

c. If a minimum efficacious dose of ruxolitinib cannot be maintained, then consider an alternative treatment modality, including participation in clinical trials. In this regard, one could consider momelotinib or pacritinib for dose-limiting anemia and thrombocytopenia, respectively (studies NCT02101268 and NCT02055781).

d. Consider splenectomy especially if severe symptoms related to splenomegaly and patient is a surgical candidate. ${ }^{31-34}$

e. The safety and efficacy of adding other drugs to ruxolitinib remains to be determined. Potential options include the addition of erythropoiesis stimulating agents or danazol (NCT01732445) for anemia and an immunomodulatory drug for thrombocytopenia. ${ }^{35,36}$

2. Late-onset cytopenias: generally reflects disease progression

a. Develops after 6-12 months of treatment at a stable dose.

b. May occur in the absence of disease progression-consider drug-drug interaction that may increase exposure to ruxolitinib (for example, concurrent voriconazole (a CYP3A4 inhibitor) treatment)

c. Cytogenetic or molecular studies may confirm clonal evolution.

d. Generally irreversible; may not respond to dose reduction or treatment interruption.

\section{Management options:}

a. Discontinue offending drug in case of interaction with ruxolitinib.

b. Exclude cumulative toxicity: if feasible, hold ruxolitinib and await blood count recovery over days to weeks.

c. Generally do not favor alternative JAK inhibitor if clonal evolution documented given the absence of selective anticlonal activity for this drug class.

d. Consider ASCT if patient is a candidate for such and a donor available.

e. If not a transplant candidate, then splenectomy especially if severe symptoms related to splenomegaly and patient is a surgical candidate. Alternative measures include participation in clinical trials although persistent cytopenias may limit study eligibility.

f. Consider adding another drug (see management of Scenario \#1[e]). 
3. Disease progression: accelerated phase disease (progressively increasing circulating blasts or monocytes, clonal evolution)

a. Generally seen in the context of worsening leukocytosis, thrombocytopenia or anemia and commonly with loss of spleen response, although disease-related symptoms may still be well controlled.

b. May meet criteria chronic myelomonocytic leukemia. Bone marrow morphologic review may show increasing blasts or dysplastic features.

c. Cytogenetic or molecular studies may confirm clonal evolution.

\section{Management options}

a. Consider discontinuing ruxolitinib (challenging decision if continuing benefit in terms of spleen/symptom response). Generally do not favor alternative JAK inhibitor (does not reduce risk of leukemic transformation).

b. Consider ASCT if patient is a candidate for such and a donor available.

c. For non-transplant candidates, individualized treatment options include single agent cladribine chemotherapy or low-dose abdominal radiotherapy. ${ }^{37}$ Hypomethylating agents (that is, 5 -azacitidine or decitabine) have limited value. ${ }^{38-42}$ Formal studies in the latter regard are ongoing (NCT02076191).

4. Loss of spleen response in the context of ruxolitinib dose reduction

a. Occurs mostly in the context of dose reduction for AEs.

b. Spleen volume changes may be dose sensitive within a relatively narrow dose range.

\section{Management options}

a. Spleen response generally regained with ruxolitinib dose increase (if feasible).

b. If minimum efficacious dose of ruxolitinib cannot be maintained, then consider alternative therapy, including other JAK inhibitors. Although anecdotal reports of efficacy exist, the value and toxicity of adding hydroxyurea or interferon-a remains to be formally studied.

5. Loss of spleen response: on a stable ruxolitinib treatment dose

a. If associated with worsening cytopenias, increasing leukocytosis and circulating blasts, evidence for clonal evolution, then disease progression is likely.

b. If not associated with above features, consider treatment noncompliance (rare), drug-drug interaction or gastrointestinal malabsorption that decreases exposure to ruxolitinib.

\section{Management options}

a. If data suggest disease progression and/or clonal evolution, then manage as Section \#3 above.

b. Check for drug-drug interaction (for example, concurrent treatment with a strong CYP3A4 inducer), malabsorption, and patient compliance with ruxolitinib.

c. If clinically efficacious dose of ruxolitinib cannot be reached (in the absence of disease progression), then consider alternative therapy including other JAK inhibitors.

6. Serious opportunistic infection: treatment-related immunosuppression

a. Ruxolitinib is immunosuppressive, potentially through inhibition of dendritic cell function. ${ }^{43,44}$

b. Severe opportunistic infections have been described, including C. neoformans and $P$. jiroveci pneumonia, ${ }^{45,46}$ T. gondii retinitis, ${ }^{47}$ disseminated tuberculosis, ${ }^{48}$ hepatitis $\mathrm{B}$ virus reactivation, ${ }^{49} H$. simplex virus reactivation ${ }^{50}$ and progressive multifocal leukoencephalopathy. ${ }^{51}$

c. The true incidence of serious infectious complications remains to be defined.

Management options (in addition to treating opportunistic infection)

a. Prevention: review screening, monitoring and primary prophylaxis strategies to mitigate risk of opportunistic infections where possible. ${ }^{44}$

b. Favor discontinuing ruxolitinib (challenging decision if continuing clinical benefit in terms of spleen/symptom response and dearth of alternative treatment options).

c. Class effect: other JAK inhibitors contraindicated?

d. Secondary prophylaxis (indefinite?) is warranted where feasible, for example, after $P$. jiroveci pneumonia.

e. Consider alternative treatment options: may be limited depending upon disease and patient status.

\section{Severe withdrawal or rebound symptoms}

a. Generally seen within hours to days of discontinuation of ruxolitinib.

b. Symptom severity may transiently exceed pre-JAK inhibitor baseline levels due to rebound effect.

c. Presentation may be highly variable (see above).

d. Predisposing factors: higher ruxolitinib dose before treatment interruption, comorbid conditions (for example, severe pulmonary hypertension).

e. Mitigating factors: tapering ruxolitinib dose; overlapping treatment with corticosteroids and/or hydroxyurea.

\section{Management options}

a. Urgent treatment with corticosteroids: high doses may be necessary initially.

b. Consider reinstituting treatment with recently discontinued ruxolitinib: consider risk to benefit ratio carefully, may be feasible if discontinuation was for 'lack of efficacy' versus serious $\mathrm{AE}$.

c. Consider starting myelosuppressive therapy (for example, hydroxyurea) especially if uncontrolled myeloproliferation is evident (for example, progressive leukocytosis and increasing blasts).

d. Intensive supportive measures: vasopressors, mechanical ventilation, blood product transfusions and so on.

\section{Leukemic transformation}

a. Very poor prognosis.

b. Development of extramedullary leukemic deposits (for example, skin) in the absence of a circulating leukemic phase has been described. ${ }^{21}$

\section{Management options}

a. Pre-leukemic phase: consideration of early ASCT if high-risk features present. ${ }^{52,53}$

b. Leukemic phase: If patient is a candidate for ASCT and a donor is available, induction chemotherapy.

c. If not an transplant candidate: consider palliative chemotherapy with cladribine or daunorubicin, investigational agents if available, or supportive care.

d. We do not recommend ruxolitinib in post-myeloproliferative neoplasms AML. ${ }^{54}$

\section{Severe abnormal bleeding}

a. If severe thrombocytopenia present: distinguish between drugrelated myelosuppression versus disease progression. 
b. If bleeding is out of proportion to thrombocytopenia: then consider coagulopathy due to liver dysfunction, DIC/ICF, acquired von Willebrand syndrome, or functional platelet defect.

c. Gl bleeding is a special situation: exclude variceal bleeding due to splanchnic venous thrombosis.

\section{Management options}

a. If severe thrombocytopenia present (and no clear evidence of disease progression): then reduce dose or hold ruxolitinib, platelet transfusion.

b. If bleeding is out of proportion to thrombocytopenia:

(i) Urgent comprehensive bleeding workup: assess for coagulation factor deficiencies, acquired von Willebrand syndrome, DIC/ICF, acquired dysfibrinogenemia, platelet function defect and so on.

(ii) Treat/correct underlying hemostatic defects: platelets, fresh frozen plasma, prothrombin complex concentrate, cryoprecipitate, von Willebrand factor concentrate, anti-fibrinolytic agents (for example, tranexamic acid), recombinant factor VIla (rarely).

(iii) Consider starting myelosuppressive therapy if uncontrolled myeloproliferation is evident: hydroxyurea, cladribine and so on.

(iv) Surgical or interventional radiology intervention for a discrete bleeding source: arterial embolization, abdominal reexploration for post-splenectomy bleeding and so on.

(v) Variceal bleeding: endoscopy with banding or sclerotherapy, $\beta$-blocker to reduce portal hypertension.

\section{CONCLUSIONS AND FUTURE DIRECTIONS}

It is becoming increasingly evident that ruxolitinib is discontinued in over half of MF patients within the first 2-3 years of starting such therapy. Consequently, there is a critical need to clarify the causes of drug discontinuation and develop a working definition of 'treatment failure', especially in the context of clinical trials to permit comparison of treatment results across studies. In this regard, we underscore the need to distinguish between (i) primary treatment resistance, (ii) sub-optimal response, (iii) disease relapse, (iv) disease progression, (v) AEs and (vi) other reasons. Notably, drug discontinuation may ensue from more than one of the aforementioned reasons and may also be confounded by 'mixed response'; the latter implies the concomitant presence of both treatment response and reasons for drug interruption/ discontinuation.

A related goal also of critical importance is to clarify the clinical outcome (including survival) as well as the response to subsequent therapies in MF patients following ruxolitinib 'treatment failure'. The correlation between specific causes of treatment failure and clinical outcome as well as response to second-line therapies may point to significant differences in the disease biology between MF patients. These observations will likely inform our therapeutic approach, in particular with regard to the aggressiveness of subsequent treatment that is selected. For example, if development of isolated late cytopenias not reversible by ruxolitinib dose reduction/treatment interruption is found to be associated with a higher risk of disease progression and/or poor survival, then it may bias toward consideration of early ASCT versus other salvage medical therapies.

The ruxolitinib discontinuation data may also drive the choice of initial therapy in certain situations. For example, in a patient with intermediate-1 risk MF with significant splenomegaly-related symptoms, ruxolitinib may be an appropriate initial choice for treatment to palliate symptoms. If however this patient is found to harbor a poor-risk molecular/mutation profile (for example, ASXL1 $+/\left(\right.$ CALR-) ${ }^{8}$ the predicted median life expectancy decreases from 6 years to 3 years. Furthermore, there is a minimum $50 \%$ probability of discontinuing ruxolitinib treatment for some reason within 2-3 years, and the efficacy of post-ruxolitinib 'failure' salvage therapies is not well established. In this context, ruxolitinib is unlikely to provide for long-term disease control and consideration of ASCT early as opposed to at time of disease relapse/progression may be warranted.

In our opinion, the '2013 IWG response criteria' and '2014 ELNIWG clinical end points for drug treatment trials' documents provide a good starting point for discussion on how to define 'true treatment failure' with ruxolitinib. ${ }^{55,56}$ For instance, primary resistance is indicated by the absence of onset of any clinical response within 28 days of starting treatment. Suboptimal response is indicated by either the failure to achieve a minimum clinical improvement response within 12 weeks or a 'mixed response' wherein supervening AEs (for example, cytopenias) complicate a clinical improvement or better clinical response, particularly if ruxolitinib dose reduction or treatment interruption is necessary. Disease relapse is indicated by loss of a previously confirmed (clinical improvement or better) clinical response or disease progression by leukemic transformation. Drug discontinuation due of other reasons would be considered separately. Ideally, post-ruxolitinib clinical trial subjects should be stratified based on the aforementioned classification so we understand which second-line therapies provide the best fit for specific clinical scenarios.

\section{CONFLICT OF INTEREST}

AT was Principal Investigator for Phase-1/2 ruxolitinib study (NCT00509899) in myelofibrosis for the Mayo Clinic (Rochester) site. The sponsor (Incyte) funded the clinical trial and provided study drug, but was not involved in any way in the current manuscript. AP and AT are/have served as Principal Investigators for other clinical trials for myelofibrosis treatment with JAK inhibitors and other agents.

\section{AUTHOR CONTRIBUTIONS}

This manuscript was conceived and written by AP and AT without the involvement of medical writers or other form of editorial support. Both approved the final version of the manuscript.

\section{REFERENCES}

1 James C, Ugo V, Le Couedic JP, Staerk J, Delhommeau F, Lacout C et al. A unique clonal JAK2 mutation leading to constitutive signalling causes polycythaemia vera. Nature 2005; 434: 1144-1148.

2 Pardanani AD, Levine RL, Lasho T, Pikman Y, Mesa RA, Wadleigh M et al. MPL515 mutations in myeloproliferative and other myeloid disorders: a study of 1182 patients. Blood 2006; 108: 3472-3476.

3 Pikman Y, Lee BH, Mercher T, McDowell E, Ebert BL, Gozo M et al. MPLW515L is a novel somatic activating mutation in myelofibrosis with myeloid metaplasia. PLoS Med 2006; 3: e270.

4 Tefferi A, Guglielmelli P, Larson DR, Finke C, Wassie EA, Pieri L et al. Long-term survival and blast transformation in molecularly-annotated essential thrombocythemia, polycythemia vera and myelofibrosis. Blood 2014; 124: 2507-2513.

5 Tefferi A, Lasho TL, Jimma T, Finke CM, Gangat N, Vaidya R et al. One thousand patients with primary myelofibrosis: the mayo clinic experience. Mayo Clin Proc 2012; 87: 25-33.

6 Gangat N, Caramazza D, Vaidya R, George G, Begna K, Schwager S et al. DIPSS plus: a refined Dynamic International Prognostic Scoring System for primary myelofibrosis that incorporates prognostic information from karyotype, platelet count, and transfusion status. J Clin Oncol 2011; 29: 392-397.

7 Guglielmelli P, Lasho TL, Rotunno G, Score J, Mannarelli C, Pancrazzi A et al. The number of prognostically detrimental mutations and prognosis in primary myelofibrosis: an international study of 797 patients. Leukemia 2014; 28: 1804-1810. 
8 Tefferi A, Guglielmelli P, Lasho TL, Rotunno G, Finke C, Mannarelli C et al. CALR and ASXL1 mutations-based molecular prognostication in primary myelofibrosis: an international study of 570 patients. Leukemia 2014; 28: 1494-1500.

9 Tefferi A, Abdel-Wahab O, Cervantes F, Crispino JD, Finazzi G, Girodon F et al. Mutations with epigenetic effects in myeloproliferative neoplasms and recent progress in treatment: Proceedings from the 5th International Post-ASH Symposium. Blood Cancer J 2011; 1: e7.

10 Tefferi A. Primary myelofibrosis: 2014 update on diagnosis, risk-stratification, and management. Am J Hematol 2014; 89: 915-925.

11 Ballen K. How to manage the transplant question in myelofibrosis. Blood Cancer J 2012; 2: e59.

12 Verstovsek S, Kantarjian H, Mesa RA, Pardanani AD, Cortes-Franco J, Thomas DA et al. Safety and efficacy of INCB018424, a JAK1 and JAK2 inhibitor, in myelofibrosis. N Engl J Med 2010; 363: 1117-1127.

13 Verstovsek S, Mesa RA, Gotlib J, Levy RS, Gupta V, DiPersio JF et al. A double-blind, placebo-controlled trial of ruxolitinib for myelofibrosis. N Engl J Med 2012; 366: 799-807.

14 Harrison C, Kiladjian JJ, Al-Ali HK, Gisslinger H, Waltzman R, Stalbovskaya V et al. JAK inhibition with ruxolitinib versus best available therapy for myelofibrosis N Engl J Med 2012; 366: 787-798.

15 Jakafi (Ruxolitinib) label. http://www.accessdata.fda.gov/drugsatfda_docs/label/2011/ 202192lbl.pdf.

16 Cervantes F, Vannucchi AM, Kiladjian JJ, Al-Ali HK, Sirulnik A, Stalbovskaya V et al. Three-year efficacy, safety, and survival findings from COMFORT-II, a phase 3 study comparing ruxolitinib with best available therapy for myelofibrosis. Blood 2013; 122: 4047-4053.

17 Verstovsek S, Mesa R, Gotlib J, Levy R, Gupta V, DiPersio J et al. Long-term outcomes of ruxolitinib therapy in patients with myelofibrosis: 3-year update from COMFORT-I. Blood 2013; 122, abstract 396.

18 Fonseca E, Silver RT, Kazis L, Usman lqbal S, Rose M, Khan N et al. Ruxolitinib discontinuation in patients with myelofibrosis: an analysis from clinical practice. Blood 2013; 122, abstract 2833.

19 Tefferi A, Litzow MR, Pardanani A. Long-term outcome of treatment with ruxolitinib in myelofibrosis. N Engl J Med 2011; 365: 1455-1457.

20 Verstovsek S, Kantarjian HM, Estrov Z, Cortes JE, Thomas DA, Kadia T et al. Long-term outcomes of 107 patients with myelofibrosis receiving JAK1/JAK2 inhibitor ruxolitinib: survival advantage in comparison to matched historical controls. Blood 2012; 120: 1202-1209.

21 Kremyanskaya M, Mascarenhas J, Rampal R, Hoffman R. Development of extramedullary sites of leukaemia during ruxolitinib therapy for myelofibrosis. Br J Haematol2014; 167: 144-146.

22 Tefferi A, Pardanani A. Serious adverse events during ruxolitinib treatment discontinuation in patients with myelofibrosis. Mayo Clin Proc2011; 86: 1188-1191.

23 Dai T, Friedman EW, Barta SK. Ruxolitinib withdrawal syndrome leading to tumor lysis. J Clin Oncol2013; 31: e430-e432.

24 Beauverd Y, Samii K. Acute respiratory distress syndrome in a patient with primary myelofibrosis after ruxolitinib treatment discontinuation. Int J Hemato/2014; 100 498-501.

25 Robin M, Francois S, Huynh A, Cassinat B, Bay J-O, Cornillon J et al. Ruxolitinib before allogeneic hematopoietic stem cell transplantation (HSCT) in patients with myelofibrosis: a preliminary descriptive report of the JAK ALLO study, a phase II trial sponsored By Goelams-FIM in collaboration With The Sfgmtc. Blood 2013; 122, abstract 306.

26 Kroger N, Alchalby H, Ditschkowski M, Wolf D, Wulf G, Zabelina T et al. Ruxolitinib as pretreatment before allogeneic stem cell transplantation for myelofibrosis. Blood 2013; 122, abstract 392.

27 Lebon D, Rubio M, Legrand F, Kiladjian J-J, Mohty M, Cahn J-Y et al. Ruxolitinib for patients with primary or secondary myelofibrosis before allogeneic hematopoietic stem cell transplantation (allo-HSCT): a retrospective study of the Société Française De Greffe De Moelle Et De Thérapie Cellulaire (SFGM-TC). Blood 2013, 122, abstract 2111

28 Barosi G, Zhang MJ, Peter Gale R. Does ruxolitinib improve survival of persons with MPN-associated myelofibrosis? Should it? Leukemia 2014; 28: 2267-2270.

29 Gale RP, Barosi G. Commentary. Leukemia Res2014; 38: 1013-1015.

30 Harrison C, Schaap N, Zweegman S, Jourdan E, Kiladjian J, Cervantes F et al. Efficacy and safety of Fedratinib (SAR302503/TG101348) in patients with intermediate- or high-risk myelofibrosis (MF), post-polycythemia vera (PV) MF, or post-essential thrombocythemia (ET) MF previously treated with Ruxolitinib: interim results from a phase II tudy (JAKARTA-2). Blood 2013; 122: abstract 661.

31 Santos FP, Tam CS, Kantarjian H, Cortes J, Thomas D, Pollock R et al. Splenectomy in patients with myeloproliferative neoplasms: efficacy, complications and impact on survival and transformation. Leuk Lymphoma 2014; 55: 121-127.
32 Mesa RA, Nagorney DS, Schwager S, Allred J, Tefferi A. Palliative goals, patient selection, and perioperative platelet management: outcomes and lessons from 3 decades of splenectomy for myelofibrosis with myeloid metaplasia at the Mayo Clinic. Cancer 2006; 107: 361-370.

33 Tefferi A, Mesa RA, Nagorney DM, Schroeder G, Silverstein MN. Splenectomy in myelofibrosis with myeloid metaplasia: a single-institution experience with 223 patients. Blood 2000; 95: 2226-2233.

34 Cervantes F. How I treat splenomegaly in myelofibrosis. Blood Cancer J 2011; 1: e37.

35 McMullin MF, Harrison C, Niederwieser D, Demuynck H, Jakel N, Sirulnik A et al. The use of erythropoietic-stimulating agents (ESAs) with ruxolitinib in patients with primary myelofibrosis (PMF), post-polycythemia vera myelofibrosis (PPV-MF), and post-essential thrombocythemia myelofibrosis (PET-MF). Blood 2012; 120, abstract 2838.

36 Grunwald MR, Spivak JL. Ruxolitinib enhances platelet production in patients with thrombocytopenic myelofibrosis. J Clin Oncol 2014; e-pub ahead of print 23 June 2014; doi:10.1200/JCO.2013.51.0057

37 Pardanani A, Brown P, Neben-Wittich M, Tobin R, Tefferi A. Effective management of accelerated phase myelofibrosis with low-dose splenic radiotherapy. Am J Hematol 2010; 85: 715-716.

38 Quintas-Cardama A, Tong W, Kantarjian H, Thomas D, Ravandi F, Kornblau S et al. A phase II study of 5 -azacitidine for patients with primary and post-essential thrombocythemia/polycythemia vera myelofibrosis. Leukemia 2008; 22: 965-970.

39 Liu Y, Tabarroki A, Billings S, Visconte V, Rogers HJ, Hasrouni E et al. Successful use of very low dose subcutaneous decitabine to treat high-risk myelofibrosis with Sweet syndrome that was refractory to 5-azacitidine. Leuk Lymphoma 2014; 55: 447-449.

40 Danilov AV, Relias V, Feeney DM, Miller KB. Decitabine is an effective treatment of idiopathic myelofibrosis. Br J Haemato/2009; 145: 131-132.

41 Mesa RA, Verstovsek S, Rivera C, Pardanani A, Hussein K, Lasho T et al. 5-Azacitidine has limited therapeutic activity in myelofibrosis. Leukemia 2009; 23: 180-182.

42 Odenike O, Godwin J, Van Besien K, Huo D, Sher D, Burke P et al. Phase II trial of low dose, subcutaneous decitabine in myelofibrosis. Blood 2008; 112 abstract 2809 .

43 Heine A, Held SA, Daecke SN, Wallner S, Yajnanarayana SP, Kurts C et al. The JAK-inhibitor ruxolitinib impairs dendritic cell function in vitro and in vivo. Blood 2013; 122: 1192-1202.

44 Heine A, Brossart P, Wolf D. Ruxolitinib is a potent immunosuppressive compound: is it time for anti-infective prophylaxis? Blood 2013; 122 3843-3844

45 Wysham NG, Sullivan DR, Allada G. An opportunistic infection associated with ruxolitinib, a novel janus kinase 1,2 inhibitor. Chest 2013; 143: 1478-1479.

46 Lee SC, Feenstra J, Georghiou PR. Pneumocystis jiroveci pneumonitis complicating ruxolitinib therapy. BMJ Case Rep 2014; e-pub ahead of print 2 June 2014; doi:10.1136/bcr-2014-204950.

47 Goldberg RA, Reichel E, Oshry LJ. Bilateral toxoplasmosis retinitis associated with ruxolitinib. N Engl J Med2013; 369: 681-683.

48 Colomba C, Rubino R, Siracusa L, Lalicata F, Trizzino M, Titone L et al. Disseminated tuberculosis in a patient treated with a JAK2 selective inhibitor: a case report. BMC Res Notes 2012; 5: 552.

49 Caocci G, Murgia F, Podda L, Solinas A, Atzeni S, La Nasa G et al. Reactivation of hepatitis $B$ virus infection following ruxolitinib treatment in a patient with myelofibrosis. Leukemia 2014; 28: 225-227.

50 Tong LX, Jackson J, Kerstetter J, Worswick SD. Reactivation of herpes simplex virus infection in a patient undergoing ruxolitinib treatment. J Am Acad Dermatol 2014 70: e59-e60.

51 Wathes R, Moule S, Milojkovic D. Progressive multifocal leukoencephalopathy associated with ruxolitinib. N Engl J Med2013; 369: 197-198.

52 Tefferi A, Pardanani A, Gangat N, Begna KH, Hanson CA, Van Dyke DL et al. Leukemia risk models in primary myelofibrosis: an International Working Group study. Leukemia 2012; 26: 1439-1441.

53 Tefferi A, Jimma $\mathrm{T}$, Gangat $\mathrm{N}$, Vaidya $\mathrm{R}$, Begna $\mathrm{KH}$, Hanson $\mathrm{CA}$ et al. Predictors of greater than $80 \%$ 2-year mortality in primary myelofibrosis: a Mayo Clinic study of 884 karyotypically annotated patients. Blood 2011; 118: 4595-4598.

54 Eghtedar A, Verstovsek S, Estrov Z, Burger J, Cortes J, Bivins C et al. Phase 2 study of the JAK kinase inhibitor ruxolitinib in patients with refractory leukemias, including postmyeloproliferative neoplasm acute myeloid leukemia. Blood 2012; 119: 4614-4618.

55 Tefferi A, Cervantes F, Mesa R, Passamonti F, Verstovsek S, Vannucchi AM et al. Revised response criteria for myelofibrosis: International Working GroupMyeloproliferative Neoplasms Research and Treatment (IWG-MRT) and European LeukemiaNet (ELN) consensus report. Blood 2013; 122: 1395-1398. 
56 Barosi G, Tefferi A, Besses C, Birgegard G, Cervantes F, Finazzi G et al. Clinical end points for drug treatment trials in BCR-ABL1-negative classic myeloproliferative neoplasms: consensus statements froim European LeukemiaNET (ELN) and Internation Working Group-Myeloproliferative Neoplasms Research and Treatment (IWG-MRT). Leukemia 2014; e-pub ahead of print 25 August 2014; doi:10.1038/leu.2014.250. (c) (i) $\Theta$ This work is licensed under a Creative Commons Attribution(cc) $\bigoplus_{\mathrm{BY}} \Theta_{\mathrm{ND}}$ NonCommercial-NoDerivs 4.0 International License. The images or other third party material in this article are included in the article's Creative Commons license, unless indicated otherwise in the credit line; if the material is not included under the Creative Commons license, users will need to obtain permission from the license holder to reproduce the material. To view a copy of this license, visit http:// creativecommons.org/licenses/by-nc-nd/4.0/ 